\title{
Emergence of mobilized colistin resistance-1 in multidrug-resistant Klebsiella pneumoniae and Escherichia coli isolates from the Henan province in China: a multicentre study
}

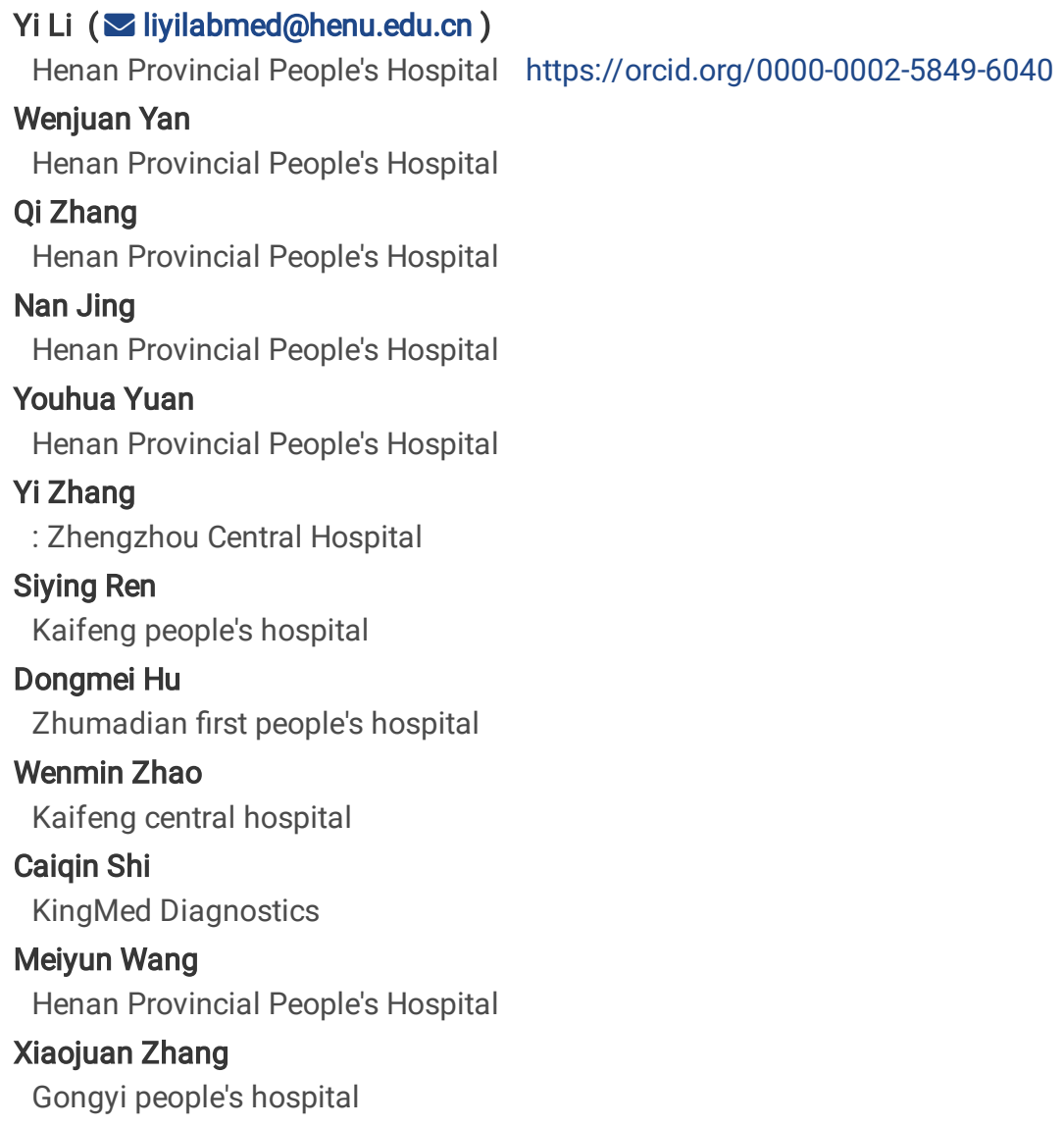

Research

Keywords: polymyxin, E. coli, K. pneumoniae, mcr-1, Henan province

Posted Date: March 16th, 2021

DOI: https://doi.org/10.21203/rs.3.rs-285784/v1

License: (c) (i) This work is licensed under a Creative Commons Attribution 4.0 International License. Read Full License 


\section{Abstract \\ Background}

The increased clinical use of polymyxin led to the emergence of polymyxin-resistant strains, especially those carrying plasmid-borne mobilized colistin resistance $(\mathrm{mcr})$ gene variants. In this study, we aimed to evaluate the prevalence and characteristics of polymyxinresistant Klebsiella pneumoniae and Escherichia coli isolates from the Henan province, China.

\section{Methods}

A total of 16 polymyxin-resistant isolates among 2301 E. coli and K. pneumoniae isolates collected in 6 local hospitals in the Henan province were studied. The isolates were identified using matrix-assisted laser desorption/ionization time-of-flight mass spectrometry, and the minimum inhibitory concentrations (MICs) were determined using the microbroth dilution technique. Polymyxin-resistant isolates were further analysed for $m c r-1$ and carbapenemase-mediated resistance using the modified carbapenem inactivation method, the ethylenediaminetetraacetic acid-modified carbapenem inactivation method, and polymerase chain reaction. Pulsed-field gel electrophoresis (PFGE) and multi-locus sequence typing (MLST) were performed to disclose the phylogenetic relationships of the polymyxin-resistant isolates. The clinical characteristics of patients infected with the polymyxin-resistant isolates were also retrospectively analysed.

\section{Results}

$5 / 1499(0.3 \%)$ and $11 / 802(1.4 \%)$ E. coli and K. pneumoniae isolates, respectively, were polymyxin-resistant. The MICs of polymyxin were in the range of $4-64 \mu \mathrm{g} / \mathrm{mL}$ and all of the 16 polymyxin-resistant isolates were susceptible to tigecycline. Additionally, four of the five $E$. coli polymyxin-resistant isolates were $m c r-1$ positive; one of them was also carbapenem-resistant, carrying $b / a_{\mathrm{NDM}-5}$. Conversely, only $1 / 11 \mathrm{~K}$. pneumoniae isolates was $m c r-1$ positive, while 9 polymyxin-resistant isolates were also carbapenem-resistant (PRCRKP), carrying $b / a_{\text {KPC-2 }}$ but not $m c r-1$. MLST results showed that the five $E$. coli isolates belonged to four sequence types (STs), including ST2, ST132, ST632, and ST983, while all PRCRKP isolates belonged to ST11. However, all 16 isolates showed different PFGE types using a genetic similarity of $\geq$ $95 \%$. Furthermore, $33.3 \%(5 / 15)$ of the patients carrying polymyxin-resistant $K$. pneumoniae isolates showed a history of polymyxin use, and 10/15 (66.7\%) patients displayed good clinical outcomes.

\section{Conclusion}

The polymyxin resistance rate of $K$. pneumoniae was slightly higher than that of $E$. coli in the Henan province; however, $m c r-1$ was only detected in one $K$. pneumoniae isolate. Thus, close monitoring is needed to prevent and control the spread of PRCRKP.

\section{Background}

Antibiotic resistance has become a global public health priority in recent years. Colistin, also known as polymyxin, is one of the few therapeutic options available for the treatment of infectious diseases caused by multidrug-resistant gram-negative bacteria [1]. In China, injectable polymyxin was approved for the treatment of bacterial infections in January 2017. However, because of the increased usage of polymyxin in clinical settings, polymyxin-resistant strains, especially those carrying plasmid-borne mobilized colistin resistance $(\mathrm{mcr})$ gene variants have emerged in China and various countries worldwide [2]. Moreover, the intraspecies transmission of resistant isolates has already been reported [1, 3].

Since its discovery in southern China in late 2015 [4], mcr-1 has spread to over 40 countries and regions, implying that it plays a prevalent role in the transferability of polymyxin resistance. Of note, $m c r-1$-positive strains have also emerged in the Henan province, including in pigderived Escherichia coli isolates [5]. In fact, clinical E. coli isolates co-producing bla $a_{\text {NDM }}$ and $m c r-1$ were previously reported by our laboratory [6], and a novel conjugative mcr-8.2-bearing plasmid was identified in an almost pan-resistant hypermucoviscous Klebsiella pneumoniae ST11 isolate [7]. However, overall, the reports of $m c r$ in human-derived $E$. coli and $K$. pneumoniae isolates are mainly centred outside of Henan.

Additionally, colistin resistance in $K$. pneumoniae can be mediated by chromosomal mutations in genes involved in lipopolysaccharide synthesis, namely $p h o P Q, p m r A B$, and $c r r A / c r r B$ as well as the $m g r B$ regulatory gene [8-10]. 
To better understand the epidemiological trends and characteristics of polymyxin-resistant clinical strains, here, we looked for polymyxin resistance among isolates collected at 6 hospitals in Henan from 2018 to 2019. A total of 16 polymyxin-resistant strains were collected, and their molecular resistance characteristics were analysed. To the best of our knowledge, this is the first multi-centre study screening for polymyxin-resistant isolates among E. coli and K. pneumoniae in the Henan province, China.

\section{Methods}

\section{Sample collection}

Non-duplicated E. coli and K. pneumoniae strains were obtained from routine microbiological cultures of clinical samples including blood, urine, sputum, bronchoalveolar lavage fluid (BAL), bile, hydrothorax, ascites, and various other specimens. A total of 2301 strains were isolated from 6 hospitals in Henan. Identification at the species level was performed by matrix-assisted laser desorption/ionization time-offlight mass spectrometry (MALDI-TOF MS; Bruker Daltonik GmbH, Bremen, Germany).

\section{Susceptibility testing and determination of the minimum inhibitory concentrations (MICs)}

Susceptibility to polymyxin was screened using Etest strips (Antu, Zhengzhou, China); only isolates with MICs higher than $2 \mu \mathrm{g} / \mathrm{mL}$ were subjected to further susceptibility testing for validation using the microbroth dilution method based on the clinical breakpoints defined by the European Committee on Antimicrobial Susceptibility Testing (EUCAST, 2018) [11].

Additionally, susceptibility to ampicillin (AMP), meropenem (MEM), imipenem (IPM), ceftazidime (CAZ), cefotaxime (CTX), cefazolin (KZ), ampicillin/sulbactam (SAM), aztreonam (ATM), cefepime (FEP), piperacillin/tazobactam (TZP), levofloxacin (LEV), amikacin (AK), gentamicin (GN), trimethoprim/sulfamethoxazole (SXT), ceftazidime/avibactam (CZA), and tigecycline (TGC) was determined only in the context of polymyxin-resistant strains using the microbroth dilution method and interpreted according to the Clinical and Laboratory Standards Institute (CLSI) guidelines [12].

\section{Multi-locus sequence typing (MLST)}

Polymyxin-resistant K. pneumonia and E. coli isolates were typed using MLST following the scheme established by the Pasteur Institute (https://bigsdb.pasteur.fr/klebsiella/klebsiella.html; https://bigsdb.pasteur.fr/ecoli/ ecoli.html).

\section{Characterization of $m c r-1$ - and carbapenemase mediated resistance}

The modified carbapenem inactivation method ( $\mathrm{mCIM}$ ) and ethylenediaminetetraacetic acid-modified carbapenem inactivation method (eCIM), which are recommended by the CLSI, were used for the phenotypic detection of carbapenemase production. The presence of carbapenem resistance genes ( $b / a_{\mathrm{VIM}}, b / a_{1 \mathrm{MP}}, b / a_{\mathrm{KPC}}, b / a_{\mathrm{NDM}}$, and $b / a_{\text {oxa-48-like }}$ ) and of the polymyxin resistance gene $m c r-1$ in polymyxinresistant isolates were screened by polymerase chain reaction using the methods described previously $[13,14]$.

\section{Pulsed-field gel electrophoresis (PFGE)}

Molecular epidemiology of all polymyxin-resistant strains was determined by PFGE after total chromosomal DNA digestion with Xbal in accordance with a previous report [15]. The PFGE patterns were analysed using the BIONUMERICS software (Applied Maths NV, Sint-MartensLatem, Belgium) using the Dice similarity coefficient. Isolates were considered as the same strain (PFGE type) if they possessed a genetic similarity of $\geq 95 \%$.

\section{Results}

\section{Overall prevalence of polymyxin-resistant strains}

Over the course of the study, 16 out of the 2301 E. coli and K. pneumoniae isolates (0.7\%) were found to be polymyxin-resistant: 5 E. coli and 11 K. pneumoniae isolates, collected from 6 different hospitals. The prevalence of polymyxin resistance in E. coli and K. pneumoniae was $0.3 \%$ and $1.4 \%$, respectively (Table 1 ).

\section{Antimicrobial susceptibility testing}

Antimicrobial susceptibility testing showed that all of the isolates (100\%) were resistant to AMP, KZ, and CTX; $93.3 \%$ were resistant to LEV; $86.6 \%$ were resistant to CAZ, FEP, and ATM; $80 \%$ were resistant to SAM and TZP; $66 \%$ were resistant to GN and AK; $62.5 \%$ were resistant to IPM and MEM; and 60\% were resistant to SXT. Only one isolate was resistant to CZA (6.3\%), and all of them were susceptible to TGC (100\%) (Figure 1). 
The MICs of polymyxin in the context of these 16 strains ranged from $4-64 \mu \mathrm{g} / \mathrm{mL}$ : the full range in the context $K$. pneumoniae isolates (median: $64 \mu \mathrm{g} / \mathrm{mL}$ ) and $4 \mu \mathrm{g} / \mathrm{mL}$ in the context of $E$. coli isolates (Table 2).

\section{Detection of antimicrobial resistance genes}

Among the 16 polymyxin-resistant isolates, 5 carried the $m c r-1$ gene, including $1 \mathrm{~K}$. pneumoniae and 4 E. coli isolates. In addition, $9 \mathrm{~K}$. pneumoniae and $1 \mathrm{E}$. coli isolates were carbapenemase-positive. The $\mathrm{mCIM}$ and eCIM results showed that the 9 K. pneumoniae isolates were serine carbapenemase-positive, and the $E$. coli isolate was metallo-carbapenemase-positive. Furthermore, the PCR results showed that the 9 K. pneumoniae isolates were bla $a_{\mathrm{KPC}-2}$-positive, but none of them carried $m c r-1$. On the other hand, the $E$. coli isolate was both $b / a_{\mathrm{NDM}-5^{-}}$and mcr-1-positive. Of note, no other carbapenemase genes, such as bla $\mathrm{IMP}_{\mathrm{MP}}, b / a_{\mathrm{VIM}}$, and bla ${ }_{\mathrm{OXA} 48-\text { like, }}$ were detected (Table 2).

\section{Epidemiological characterization}

MLST analysis showed that the nine K. pneumoniae carbapenem-resistant isolates all belonged to sequence type (ST) 11. On the other hand, among the five $E$. coli isolates, two belonged to ST132『and the other three belonged to ST2, ST983, and ST632 (Table 2).

Of note, dendrogram analysis of PFGE at $95 \%$ similarity revealed that homology among the 5 E. coli and 11 K. pneumoniae isolates was low and sporadic, suggesting a very low likelihood of clonal spread (Figure 2).

\section{Clinical characteristics of the patients infected with polymyxin-resistant isolates}

The 16 polymyxin-resistant isolates were collected within 1 year from 15 patients aged 2 months to 93 years old, in 6 hospitals. Two strains were isolate from a single patient, from blood and urine samples, while each of the other 14 patients showed only 1 strain, isolated from urine $(n=4)$, BAL $(n=3)$, blood $(n=3)$, secretion $(n=2)$, peritoneal puncture fluid $(n=1)$ and sputum $(n=1)$ samples. The underlying diseases in these patients included cerebrovascular disease $(n=3)$, urinary tract disease $(n=3)$, pneumonia $(n=2)$, sepsis $(n=1)$, fever $(n=1)$, acute coronary syndrome $(n=1)$, pregnancy-induced hypertension $(n=1)$, premature baby $(n=1)$, infection around the prosthesis $(n=1)$, and Guillain-Barre syndrome $(n=1)$. Of note, five patients received polymyxin treatment before the isolation of polymyxin-resistant strains. Importantly, ten patients displayed positive clinical outcomes (Table 3).

\section{Discussion}

Polymyxin has been used against aggressive infections caused by multidrug-resistant bacteria; however, its use has been severely compromised by the emergence of plasmid-mediated polymyxin resistance in Enterobacteriaceae. Hence, in this study, we surveyed the polymyxin resistance rates in E. coli and K. pneumoniae isolates from hospitalized patients at six local hospitals in the Henan province, China.

Among the total $2301 \mathrm{E}$. coli and K. pneumoniae isolates, $16(0.7 \%)$ strains were polymyxin-resistant, 5 of which carried the $m c r-1$ gene. Of note, of the 1499 E. coli isolates, 5 (0.3\%) were polymyxin-resistant and 4 were $m c r-1$-positive; on the other hand, of the 802 K. pneumoniae isolates, 11 (1.4\%) were polymyxin-resistant, 1 of which carried $m c r-1$. Previously, $0.88 \%(34 / 3854)$ of the E. coli isolates and $0.21 \%$ (5/2410) of the K. pneumoniae isolates carrying mcr-1 were reported in the China Antimicrobial Resistance Surveillance Trial [16]. Additionally, another study found that $1 \%(20 / 1495)$ of the $E$. coli isolates and $0.18 \%(1 / 571)$ of the $K$. pneumoniae isolates recovered from bloodstream infections in China were $m c r-1$-positive [17]. Our results are, therefore, in line with the previous ones, with rates not exceeding $1.5 \%$. Although $m c r-1$ was more common in E. coli isolates than in K. pneumoniae isolates, the polymyxin resistance rate of $K$. pneumoniae was slightly higher than that of $E$. coli in our study, which is presumably due to antibiotic selection because the detection rate (32.8\%) of carbapenem-resistant $K$. pneumoniae (PRCRKP) in Henan ranked first among all Chinese provinces in 2019 (http://www.carss.cn/Report/Details?ald=770). Of note, compared to polymyxin-resistant $E$. coli, polymyxin-resistant $K$. pneumoniae were associated with 8-64 times higher MICs, suggesting chromosomal mutations in related genes, such as $p h o P / p h o Q, p m r A / p m r B$, and $m g r B$. Additionally, other intrinsic mechanisms might also play important roles in increasing polymyxin resistance in $K$. pneumoniae [17].

Two carbapenemase genes, $b / a_{\mathrm{KPC}}$ and $b / a_{\mathrm{NDM}}$, are responsible for the phenotypic resistance of $90 \%$ of carbapenem-resistant Enterobacteriaceae strains in China [18]. The co-existence of $m c r$ and carbapenemase genes, such as b/a $a_{\mathrm{NDM}-5}$ [19], bla ${ }_{\mathrm{NDM}-4}$ [20], b/a ${ }_{\mathrm{KPC}}[21]$, and bla $a_{\text {XXA }}$ [22], has been sporadically reported in different countries. In the national monitoring data from China, one report showed that the mcr-1 gene was detected in 4.6\% (13/282) of carbapenem-resistant E. coli isolates and coexisted with the New Delhi metallo-enzyme (NDM)-5 in one strain [23]. In another study, the mcr-1 prevalence among carbapenem-resistant $E$. coli and PRCRKP isolates was 3.7\% (14/376) and $0 \%(0 / 1134)$, respectively, and 14 carbapenem-resistant $E$. coli isolates coproduced b/a ${ }_{\mathrm{NDM} 4 / 5 / 9}$ with $m c r-1$ [24]. In this study, only one $E$. coli isolate coproduced $m c r-1$ and $b / a_{\mathrm{NDM}-5}$. 
An E. coli isolate belonging to ST167 that co-expressed bla NDM $_{\text {and }}$ mcr-1 was previously reported in Henan [6, 25], but in our study, the aforementioned coproducing E. coli isolate belonged to ST2. The other E. coli strains in this study belonged to ST132, ST983, and ST632. Additionally, nine PRCRKP isolates belonged to ST11, but PFGE showed different types, suggesting these strains, all isolated from the same hospital, were unrelated. Altogether, our results demonstrate that polymyxin-resistant isolates are non-clonal and have different virulence and resistance potentials.

The patients carrying polymyxin-resistant isolates had varying severities of illness, and $33.3 \%$ of them had a history of polymyxin use. Moreover, $66.7 \%$ of them were cured, and these positive outcomes could be explained by the finding that most polymyxin-resistant isolates remained susceptible to other antimicrobials, such as CZA, SXT, and TGC.

In conclusion, here, we show that polymyxin resistance rate of $K$. pneumoniae is slightly higher than that of $E$. coli, while the presence of $m c r-$ 1 is lower in polymyxin-resistant $K$. pneumoniaeversus $E$. coli in the Henan province, China. Further molecular investigations and studies are warranted to elucidate the polymyxin resistance mechanism of PRCRKP. In addition, continuous and close monitoring is required to prevent the dissemination of polymyxin-resistant K. pneumoniae and E. colistrains.

\section{Abbreviations}

polymyxin and carbapenem resistant K. pneumoniae (PRCRKP), Minimum inhibitory concentrations (MICs), pulsed-field gel electrophoresis (PFGE), multi-locus sequence typing (MLST), sequence types (STs), mobilized colistin resistance (mcr), bronchoalveolar lavage fluid (BAL), ampicillin (AMP), meropenem (MEM), imipenem (IPM), ceftazidime (CAZ), cefotaxime (CTX), cefazolin (KZ), ampicillin/sulbactam (SAM), aztreonam (ATM), cefepime (FEP), piperacillin/tazobactam (TZP), levofloxacin (LEV), amikacin (AK), gentamicin (GN), trimethoprim/sulfamethoxazole (SXT), ceftazidime/avibactam (CZA), tigecycline (TGC), carbapenem inactivation method (mCIM), ethylenediaminetetraacetic acid-modified carbapenem inactivation method (eCIM)

\section{Declarations}

\section{Ethics approval and consent to participate}

This study was approved by the Ethics Committee of the Henan Provincial People's Hospital, Henan, China (20190050). The requirement for informed consent from patients was waived.

\section{Consent for publication}

No personally identifiable information was collected in this study.

\section{Availability of data and materials}

All data generated or analysed during this study are included in this published article.

\section{Competing interests}

The authors declare that they have no competing interests.

\section{Funding Information}

This work was supported by the Science and Technology Research Project of Henan Province [grant number SBGJ2018084 and 202102310355], the Joint Program of Medical Science and Technology Research of Henan Province (LHGJ20190611) and the Collaborative Innovation Major Project of Zhengzhou [grant number 20XTZX05015]. The funding agencies had no role in study design, data collection and analysis, decision to publish, or preparation of the manuscript.

\section{Authors' contributions}

WJY and YL contributed to conception and design of the article. YZ, SYR, DMH, WMZ, CQS and XJZ were responsible for microbiological tests. NJ and YHY carried out the molecular genetic studies. WJY and QZ wrote the first version of the manuscript. MYW and YL conceived and supervised the project. All authors critically revised the manuscript, and read and approved the final manuscript.

\section{Acknowledgments}

We would like to thank Editage (www.editage.cn) for English language editing. 


\section{References}

[1] Sun J, Zhang H, Liu YH, Feng Y. Towards understanding MCR-like colistin resistance. Trends Microbiol. 2018;26(9):794-808.

[2] Giamarellou H. Epidemiology of infections caused by polymyxin-resistant pathogens. Int J Antimicrob Agents. 2016;48(6):614-621.

[3] Nordmann P, Poirel L. Plasmid-mediated colistin resistance: an additional antibiotic resistance menace. Clin Microbiol Infect. 2016;22(5):398-400.

[4] Liu YY, Wang Y, Walsh TR, Yi LX, Zhang R, Spencer J, et al. Emergence of plasmid-mediated colistin resistance mechanism MCR-1 in animals and human beings in China: a microbiological and molecular biological study. Lancet Infect Dis. 2016;16(2):161-168.

[5] Li X, Liu B, Dong P, Li F, Yuan L, Hu G. The prevalence of mcr-1 and resistance characteristics of Escherichia coli isolates from diseased and healthy pigs. Diagn Microbiol Infect Dis. 2018; 91(1):63-65.

[6] Li Y, Sun Q, Shen Y, Zhang Y, Yang JW, Shu LB, et al. Rapid Increase in prevalence of carbapenem-resistant Enterobacteriaceae (CRE) and emergence of colistin resistance gene mcr-1 in CRE in a hospital in Henan, China. J Clin Microbiol. 2018;56(4):e01932-17.

[7] Qin S, Zhang C, Schwarz S, Li L, Dong H, Yao H, et al. Identification of a novel conjugative mcr-8.2-bearing plasmid in an almost panresistant hypermucoviscous Klebsiella pneumoniae ST11 isolate with enhanced virulence. J Antimicrob Chemother. 2020;75(9):2696-2699.

[8] Hamel M, Chatzipanagiotou S, Hadjadj L, Petinaki E, Papagianni S, Charalampaki N, et al. Inactivation of mgrB gene regulator and resistance to colistin is becoming endemic in carbapenem-resistant Klebsiella pneumoniae in Greece: A nationwide study from 2014 to 2017. Int J Antimicrob Agents. 2020;55(4):105930.

[9] Pitt, ME, Elliott, AG, Cao, MD, Ganesamoorthy D, Karaiskos I, Giamarellou H, et al. Multifactorial chromosomal variants regulate polymyxin resistance in extensively drug-resistant Klebsiella pneumoniae. Microb Genom. 2018;4(3):e000158.

[10] Poirel L, Jayol A, Bontron S, Villegas MV, Ozdamar M, Türkoglu S, et al. The mgrB gene as a key target for acquired resistance to colistin in Klebsiella pneumoniae. J Antimicrob Chemother. 2015;70(1):75-80.

[11] EUCAST. Breakpoint tables for interpretation of MICs and zone diameters. 2018.

[12] Clinical and Laboratory Standards Institute. Performance standards for antimicrobial susceptibility testing. 2018; 28th informational supplement.

[13] Dallenne C, da Costa A, Decré D, Favier C, Arlet G. Development of a set of multiplex PCR assays for the detection of genes encoding important beta-lactamases in Enterobacteriaceae. J Antimicrob Chemother. 2010;65(3):490-495.

[14] Queenan AM, Bush K. Carbapenemases: the versatile beta-lactamases. Clin Microbiol Rev. 2007;20(3):440-458.

[15] He J, Jia X, Yang S, Xu X, Sun K, Li C, et al. Heteroresistance to carbapenems in invasive Pseudomonas aeruginosa infections. Int J Antimicrob Agents. 2018;51(3):413-421.

[16] Liu X, Wang Y, Cui L, Li Y, Xue F, Liu J, et al. A retrospective study on mcr-1 in clinical Escherichia coli and Klebsiella pneumoniae isolates in China from 2007 to 2016. J Antimicrob Chemother. 2018;73(7):1786-1790.

[17] Quan J, Li X, Chen Y, Jiang Y, Zhou Z, Zhang H, et al. Prevalence of mcr-7 in Escherichia coli and Klebsiella pneumoniae recovered from bloodstream infections in China: a multicentre longitudinal study. Lancet Infect Dis. 2017;17(4):400-410.

[18] Zhang R, Liu L, Zhou H, Chan EW, Li J, Fang Y, et al. Nationwide surveillance of clinical carbapenem-resistant Enterobacteriaceae (CRE) strains in China. EBioMedicine. 2017;19:98-106.

[19] Shen Z, Hu Y, Sun Q, Hu F, Zhou H,Shu L, et al. Emerging carriage of NDM-5 and MCR-1 in Escherichia coli from healthy people in multiple regions in China: a cross sectional observational study. EClinicalMedicine. 2018;6:11-20.

[20] Le L, Tran LK, Le-Ha TD, Tran BP, Le-Vo HN, Nguyen YN, et al. Coexistence of plasmid-mediated $m c r-1$ and $b / a_{\text {NDM-4 }}$ genes in a clinical strain In Vietnam. Infect Drug Resist. 2019;12:3703-3707.

[21] Park JY, Heo ST, Kwon KT, Song DY, Lee KJ, Choi JA. MCR1 and KPC2 co-producing Klebsiella pneumoniae bacteremia: first case in Korea. Infect Chemother. 2019;51(4):399-404.

Page 6/9 
[22] Nukui Y, Ayibieke A, Taniguchik M, Aiso Y, Shibuya Y, Sonobe K, et al. Whole-genome analysis of EC129, an NDM-5-, CTX-M-14-, OXA-10and MCR-1-co-producing Escherichia coli ST167 strain isolated from Japan. J Glob Antimicrob Resist. 2019;18:148-150.

[23] Wang Q, Wang X, Wang J, Ouyang P, Jin C, Wang R, et al. Phenotypic and genotypic characterization of carbapenem-resistant Enterobacteriaceae: data from a longitudinal large-scale CRE study in China (2012-2016). Clin Infect Dis. 2018;67:S196-S205.

[24] Huang H, Dong N, Shu L, Lu J, Sun Q, Chan EWC, et al. Colistin-resistance gene mcrin clinical carbapenem-resistant Enterobacteriaceae strains in China, 2014-2019. Emerg Microbes Infect. 2020;9(1):237-245.

[25] Xu L, Wang P, Cheng J, Qin S, Xie W. Characterization of a novel b/a ${ }_{\mathrm{NDM}-5}$-harboring IncFIl plasmid and an mcr-7-bearing Incl2 plasmid in a single Escherichia coliST167 clinical isolate. Infect Drug Resist. 2019;12:511-519.

\section{Tables}

Table 1. Prevalence of polymyxin-resistant isolates in the six participating hospitals

\begin{tabular}{llll}
\hline & Hospital & No. of isolates & No. of polymyxin-resistant isolates (\%) \\
\hline Escherichia coli & & & $3(0.9)$ \\
& Hospital 1 & 326 & $1(0.4)$ \\
& Hospital 2 & 231 & $1(0.1)$ \\
Total & Hospital 6 & 942 & $5(0.3)$ \\
\hline Klebsiella pneumoniae & & 1499 & \\
& Hospital 3 & 141 & $1(0.7)$ \\
& Hospital 4 & 133 & $1(0.8)$ \\
& Hospital 5 & 78 & $2(2.6)$ \\
Total & Hospital 6 & 450 & $7(1.6)$ \\
\hline Overall total & 802 & $11(1.4)$ \\
\hline
\end{tabular}

Table 2 Phenotypic and genotypic characteristics of the polymyxin-resistant strains

\begin{tabular}{llllllll}
\hline e & Isolate & Polymyxin MIC $(\mu \mathrm{g} / \mathrm{mL})$ & mCIM & eCIM & KPC & NDM & mcr-1 \\
\hline spital5 & Kpn1 & 4 & + & - & KPC-2 & - & - \\
spital4 & Kpn2 & 32 & + & - & KPC-2 & - & - \\
spital6 & Kpn3 & 64 & + & - & KPC-2 & - & - \\
spital5 & Kpn4 & 8 & + & - & KPC-2 & - & - \\
spital6 & Kpn5 & 64 & ND & ND & - & - & mcr-1 \\
spital3 & Kpn6 & 64 & ND & ND & - & - & - \\
spital6 & Kpn7 & 16 & + & - & KPC-2 & - & - \\
spital6 & Kpn8 & 32 & + & - & KPC-2 & - & - \\
spital6 & Kpn9 & 64 & + & - & KPC-2 & - & - \\
spital6 & Kpn10 & 64 & + & - & KPC-2 & - & - \\
spital6 & Kpn11 & 64 & + & - & KPC-2 & - & - \\
spital1 & Eco1 & 4 & + & + & - & NDM-5 & mcr-1 \\
spital1 & Eco2 & 4 & ND & ND & - & - & mcr-1 \\
spital1 & Eco3 & 4 & ND & ND & - & - & mcr-1 \\
spital2 & Eco4 & 4 & ND & ND & - & - & mcr-1 \\
spital6 & Eco5 & 4 & ND & ND & - & - & - \\
\hline
\end{tabular}

Kpn, Klebsiella pneumoniae; Eco, Escherichia coli; ND, data was not collected; MIC, minimal inhibitory concentration; mCIM, modified carbapenem inactivation method; eCIM, ethylenediaminetetraacetic acid-modified carbapenem inactivation method; KPC-2, $K$. pneumoniae carbapenemase-2; NDM-5, New Delhi metallo-enzyme-5; mcr-1, mobilized colistin resistance-1

Table 3 Clinical characteristics of the patients carrying polymyxin-resistant isolates 


\begin{tabular}{|c|c|c|c|c|c|c|c|c|}
\hline Patient & $\begin{array}{l}\text { Gender/age } \\
\text { (years) }\end{array}$ & Isolate & Source & $\begin{array}{l}\text { Clinical } \\
\text { diagnosis }\end{array}$ & $\begin{array}{l}\text { Underlying } \\
\text { disease }\end{array}$ & $\begin{array}{l}\text { Indwelling } \\
\text { devices }\end{array}$ & $\begin{array}{l}\text { Antimicrobial use within } 30 \\
\text { days prior to culture }\end{array}$ & Outcome \\
\hline 1 & Male/87 & Kpn & Blood & Cerebral & Diabetes & Tracheal & TGC, Carbapenems & Discharge \\
\hline 2 & Female/33 & Kpn & Secretion & $\begin{array}{l}\text { Pneumonia, } \\
\text { AFE }\end{array}$ & No & No & $\begin{array}{l}\text { Clindamycin, } \\
\text { Quinolones }\end{array}$ & Discharge \\
\hline 3 & Male/38 & Kpn & Sputum & Septic shock & No & $\begin{array}{l}\text { CVC, } \\
\text { Tracheal }\end{array}$ & $\beta$-lactam, Quinolones & Die \\
\hline 4 & Female/69 & Eco/Eco & Blood/urine & $\begin{array}{l}\text { Acute } \\
\text { coronary }\end{array}$ & Diabetes, CHD & & $\beta$-lactam, Quinolones & Discharge \\
\hline 5 & Male/81 & Eco & Blood & Fever & Haemodialysis & No & $\beta$-lactam, Quinolones, & Discharge \\
\hline 6 & Male/93 & Eco & BAL & $\begin{array}{l}\text { Cerebral } \\
\text { haemorrhage }\end{array}$ & Diabetes & $\begin{array}{l}\text { CVC, } \\
\text { Tracheal }\end{array}$ & Carbapenems & Discharge \\
\hline 7 & Female/69 & Kpn & Urine & $\begin{array}{l}\text { Urinary } \\
\text { retention }\end{array}$ & No & CVC & $\beta$-lactam & Get worse \\
\hline 8 & Male/62 & Kpn & BAL & $\begin{array}{l}\text { Cerebral } \\
\text { haemorrhage }\end{array}$ & No & Tracheotomy & TGC, Polymyxin & Get worse \\
\hline 9 & Female/89 & Eco & $\begin{array}{l}\text { Urethral } \\
\text { secretions }\end{array}$ & cUTI & $\begin{array}{l}\text { Hypertension, } \\
\text { CHD }\end{array}$ & $\begin{array}{l}\text { Urethral } \\
\text { catheter }\end{array}$ & ß-lactam, Quinolones & Die \\
\hline 10 & Male/56 & Kpn & Urine & $\begin{array}{l}\text { Guillain- } \\
\text { Barre } \\
\text { syndrome }\end{array}$ & Hypertension & $\begin{array}{l}\text { Tracheal } \\
\text { cannula }\end{array}$ & $\begin{array}{l}\text { TGC, Polymyxin, } \\
\text { Carbapenems }\end{array}$ & Discharge \\
\hline 11 & Male/67 & Kpn & Urine & $\begin{array}{l}\text { Urethral } \\
\text { injury }\end{array}$ & Hypertension & $\begin{array}{l}\text { Urethral } \\
\text { catheter }\end{array}$ & $\beta$-lactam & Discharge \\
\hline 12 & $\begin{array}{l}\text { Female/2 } \\
\text { months }\end{array}$ & Kpn & BAL & $\begin{array}{l}\text { Premature } \\
\text { baby }\end{array}$ & No & $\begin{array}{l}\text { Tracheal } \\
\text { cannula }\end{array}$ & $\begin{array}{l}\text { Polymyxin, Fosfomycin, } \\
\text { fluconazole }\end{array}$ & Discharge \\
\hline 13 & Female/28 & Kpn & $\begin{array}{l}\text { Peritoneal } \\
\text { puncture } \\
\text { fluid }\end{array}$ & $\begin{array}{l}\text { Pregnancy- } \\
\text { induced } \\
\text { hypertension }\end{array}$ & SLE & $\begin{array}{l}\text { Peritoneal } \\
\text { drainage } \\
\text { tube }\end{array}$ & $\begin{array}{l}\text { TGC, Polymyxin, } \\
\text { Carbapenems }\end{array}$ & Discharge \\
\hline 14 & Male/89 & Kpn & Blood & $\begin{array}{l}\text { Severe } \\
\text { pneumonia }\end{array}$ & No & $\begin{array}{l}\text { Tracheal } \\
\text { cannula }\end{array}$ & $\begin{array}{l}\text { Polymyxin, } \\
\text { Carbapenems, } \\
\text { fluconazole }\end{array}$ & Die \\
\hline 15 & Female/54 & Kpn & Secretion & $\begin{array}{l}\text { Infection } \\
\text { around the } \\
\text { prosthesis }\end{array}$ & No & No & $\begin{array}{l}\text { Carbapenems, } \\
\text { Quinolones }\end{array}$ & Discharge \\
\hline
\end{tabular}

Kpn, Klebsiella pneumoniae; Eco, Escherichia coli; CHD, coronary heart disease; SLE, systemic lupus erythematosus; AFE, amniotic fluid embolism; CVC, central venous catheter; BAL, bronchoalveolar lavage fluid; cUTI, complicated urinary tract infection; TGC, tigecycline.

\section{Figures}

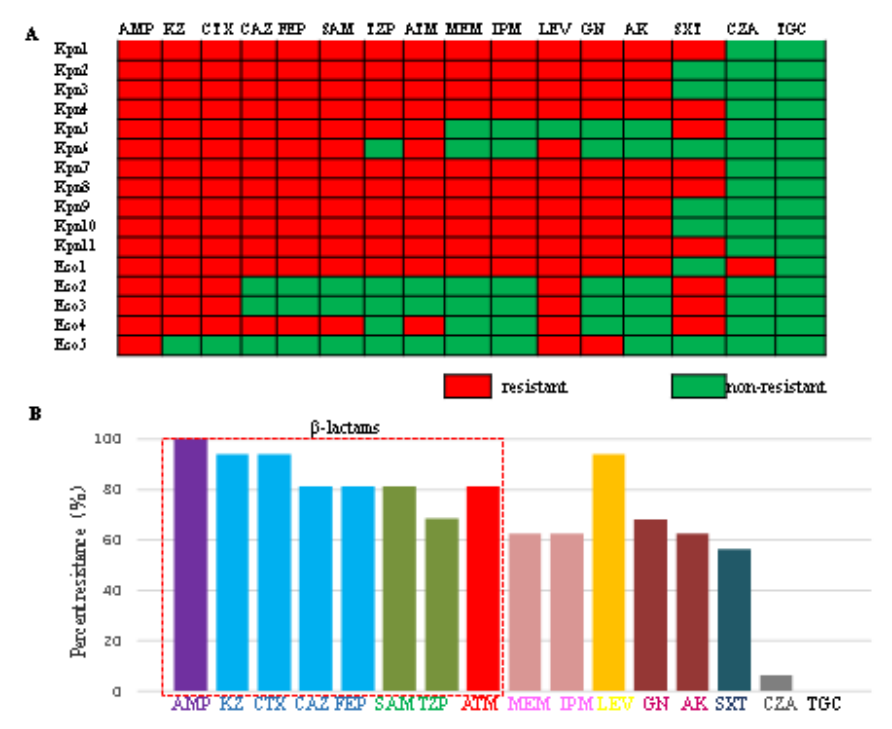

Figure 1

Antimicrobial susceptibility profiles of the 16 polymyxin-resistant isolates. (A) Heatmap showing the resistance phenotypes of each of the isolates. (B) Percentage of strains resistant to the tested antibiotics. AMP, ampicillin; KZ, cefazolin; CTX, cefotaxime; CAZ, ceftazidime; FEP, cefepime; SAM, ampicillin/sulbactam; TZP, piperacillin/tazobactam; ATM, aztreonam; MEM, meropenem; IPM, imipenem; LEV, levofloxacin; GN, gentamicin; AK, amikacin; SXT, trimethoprim/sulfamethoxazole; CZA, ceftazidime Avibactam; TGC, tigecycline. 
SampleID PFGE Hospital Carbopenemase ST

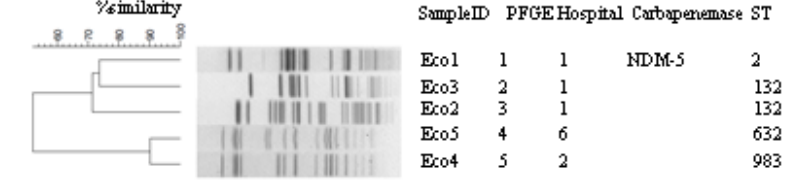

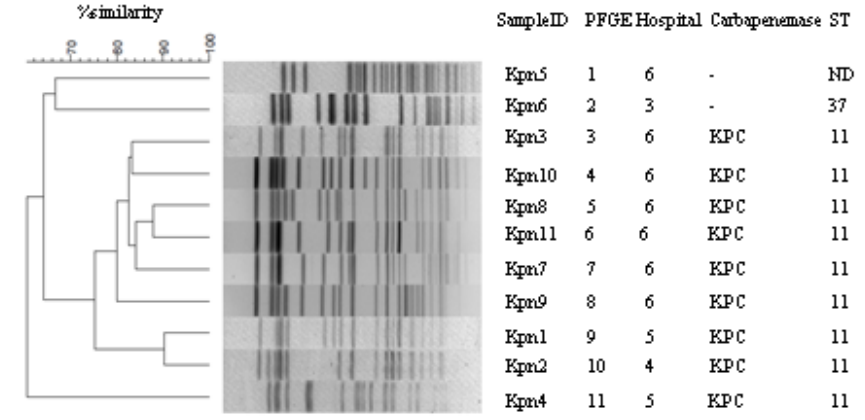

Figure 2

Pulsed-field gel electrophoresis (PFGE)-based dendrogram of polymyxin-resistant Escherichia coli (A) and Klebsiella pneumoniae (B) strains. Kpn, K. pneumoniae; Eco, E. coli; NDM-5, New Delhi metallo-enzyme-5; ST, sequence type; KPC, K. pneumoniae carbapenemase. 